\title{
SYNTHESIS, CHARACTERIZATION AND EVALUATION OF ANTI-MICROBIAL ACTIVITY OF NOVEL SUBSTITUTED QUINAZOLINE DERIVATIVES
}

\author{
P. Chaitanya ${ }^{1}$, Guguloth Ravi ${ }^{2, *}$, S. Damodhar ${ }^{1}$ and A. RavinderNath ${ }^{1}$ \\ ${ }^{1}$ Department of Pharmacy and Biotechnology, University College Technology, \\ Osmania University, Hyderabad-500007,(TS), India. \\ ${ }^{2}$ Department of Chemistry, Osmania University, Hyderabad-50000(TS),India. \\ *E-mail:drraviguguloth@gmail.com
}

\begin{abstract}
A series of novel substituted 8-bromo-2-(dimethylamino)-3-(3-(dimethylamino) propyl) quinazoline-4(3H)-one derivatives $\mathbf{2}(\mathbf{a}-\mathbf{h})$ were prepared with excellent yields and evaluate their antimicrobial activity. These compounds $\mathbf{2}(\mathbf{a}-\mathbf{h})$ are prepared the by the reactions between 8-bromo-3-(2-(dimethylamino) ethyl)-2-(methylthio) quinazoline$4(3 \mathrm{H})$-one and secondary amines. These all compounds characterization done by different spectroscopic methods PMR, IR, CMR, Mass Spectral and elemental data. All compounds were evaluated for their biological activity i.e., antibacterial and anti-fungal activity. The anti-bacterial activities were evaluated in vitro method. The antibacterial activity 8-bromo-2-(dimethylamino)-3-(3-(dimethylamino) propyl) quinazoline-4(3H)-one derivatives 2 (a-h) was assessed against three representative Gram-positive bacteria viz. Bacillus subtilis, Bacillus sphaericus and Staphylococcus aureus, and three Gram-negative bacteria viz. Pseudomonas aeruginosa, Klebsiella aerogenes and Chromobacterium violaceum, and antifungal activity against Candida albicans, Aspergillus fumigates, Trichophyton rubrum and Trichophyton mentagrophytes. Among all these compounds most of the compounds were exhibit more potent anti-bacterial and anti-fungal activity against standard drugs.

Keywords: Synthesis, 8-bromo-2-(dimethylamino)-3-(3-(dimethylamino)propyl)quinazolin-4(3H)-one derivatives, Anti-microbial activity.
\end{abstract}

(C) RASĀYAN. All rights reserved

\section{INTRODUCTION}

Past works report that so many heterocyclic compounds consist so many numbers of biological activity ${ }^{1-8}$ due to those cycles containing heteroatoms. Anti-bacterial, anti-fungal, anti-inflammatory ${ }^{9-14}$. It is a multiscale response to stress required for repair and regeneration after injury ${ }^{15-17}$. Many paths physiological path-ways like cytokines, interleukin, NF-kB, protein kinases (Adenosine Monophosphate-activated protein Kinase-AMPK), tyrosine kinases and various immunological responses regulate and mediate the process of inflammation ${ }^{18-21}$. In this process, prostaglandin synthesis is a vital step where cyclooxygenase 2 (COX-2) enzyme is one of the two key enzymes. In the second step, COX reduces PGG2 to PGH2 $2^{22,24}$. COX is majorly involved in the process of inflammation and is the targeted protein for most of the NSAIDs (non-steroidal anti-inflammatory drugs). It is also found to be a key protein in various physiological processes like gastric secretions ${ }^{25}$ gastrointestinal motility and in other pathological conditions like inflammation, arthritis and colon cancer $^{26}$. Quinazolinone derivatives were previously reported as inhibitors of various enzymes involved in process of inflammation (COX, prostaglandin E2 $)^{27}$, allergic reactions (Histamine $\mathrm{H}_{3}$ receptor inhibition) ${ }^{28}$, and also in the tumor-suppressing process through interacting with DNA, tubulin and thus acting as anticancer agents ${ }^{29}$. Some series of Quinazolinone derivatives were also shown to have remarkable antimicrobial and antifungal properties ${ }^{30}$. In addition, molecules with Quinazolinone scaffold act as regulators of calcium and sodium at cellular membranes by inhibiting sodium/calcium exchange process ${ }^{31}$.

Rasayan J. Chem., 11(3), 1357-1365(2018)

http://dx.doi.org/10.31788/RJC.2018.1131599

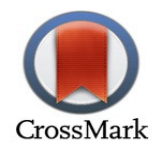


Our interests in finding novel substituted quinazolinone leads with anti-microbial activity led to the design and synthesis of 8-Bromo-2-(dimethylamino)-3-(3-(dimethylamino) propyl) quinazoline-4(3H)one derivatives. In the present investigation, we have to synthesized and evaluate tri-substituted quinazolinone derivatives as anti-microbial agents. Rationale behind the design and development of 8bromo-2-(dimethylamino)-3-(3-(dimethylamino) propyl) quinazoline-4(3H)-one derivatives is to increase overall biological activity and in order to maintain the efficacy of the molecules, alkyl linked amino dialkyl groups were introduced at $\mathrm{R}_{1}$ and aromatic /secondary amine systems were substituted at $\mathrm{R}_{2}$. These substitutions are expected to increase the hydrophobic character. Further, we are also attentive to identify the possible efficient for the respective anti-bacterial and anti-fungal activity.

\section{EXPERIMENTAL}

\section{Material and Method}

In this experiment, all reagents are used analytical reagent grade obtained from Sigma-Aldrich, Merck, SD fine and avra chemicals. With using standard procedures we purified Water, methanol, acetone, ether etc. 6- benzyl-3-(5-methyl-1-(naphthalene-1-yl)-1H-1,2,3-triazole-4-yl)-7H-[1,2,4] triazolo [3,4b] $[1,3,4]$ thiadiazine compounds ${ }^{1} \mathrm{H}$ NMR and ${ }^{13} \mathrm{C}$ NMR spectra were recorded on Bruker $300 \mathrm{MHz} \mathrm{NMR}$ instrument using tetra methyl silane (TMS).as internal standard compound and coupling constants $(J)$ are reported in $\mathrm{Hz}$ units. IR spectra of the compounds were recorded using $\mathrm{KBr}$ pellets in the range 4000-600 $\mathrm{cm}^{1}$ on Perkin-Elmer Infrared model 337. The percentage composition of C, H, N of the compounds was determined by using micro analytical techniques on Perkin Elmer 240C (USA) elemental analyzer. All reactions were monitored by thin-layer chromatography (TLC) on pre-coated silica gel F254 plates from Merck, and compounds visualized either by exposure to UV light.

\section{General Procedure}

Preparation of methyl 3-bromo-2-(methylthiocarbonothioyl)benzoate (II)

To a vigorously stirring solution of mono bromo anthranilic acid ; 0.01 mole) in dimethyl sulfoxide (20 $\mathrm{ml})$ sodium hydroxide $(1.2 \mathrm{ml} ; 20 \mathrm{~N})$ and carbon disulfide ( 0.015 moles) were added alternately, dropwise over a period of $30 \mathrm{~min}$. After complete addition, the solution was stirred for another $30 \mathrm{~m}$ and was cooled to less than $15^{\circ} \mathrm{C}-20^{\circ} \mathrm{C}$ using a freezing mixture. To this solution dimethyl sulfate $(0.01$ mole) was added dropwise for $30 \mathrm{~min}$. The solution was stirred for 3-4 hr at room temperature. The resulting mixture was poured onto crushed ice $(100 \mathrm{ml})$ with stirring. The product was extracted with chloroform $(4 \mathrm{x} 20$ $\mathrm{ml}$ ), which on evaporation yielded a yellow resinous product. This crude product was used for the next step without purification ${ }^{32}$.

IR $\left(\mathrm{cm}^{-1}\right) \quad v_{\max }: 3353.2,3056.4,2960,1715.4,1670,1645.7,1070.8,783.2,586.4,{ }^{1} \mathrm{H}$ NMR (DMSO-d 6,300 MHz) $\delta: 2.0\left(\mathrm{~s}, 3 \mathrm{H},-\mathrm{SCH}_{3}\right), 3.88\left(\mathrm{~s}, 3 \mathrm{H},-\mathrm{OCH}_{3}\right), 4.0(\mathrm{~s}, 1 \mathrm{H}, \mathrm{NH}), 7.4-7.9(\mathrm{~m}, J=8.4 \mathrm{~Hz}, 3 \mathrm{H}, \mathrm{Ar}-\mathrm{H}),,{ }^{13} \mathrm{C} \quad \mathrm{NMR}$

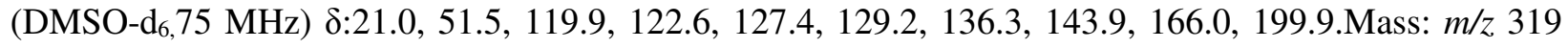
$\left(\mathrm{M}^{+}\right), 321(\mathrm{M}+2)$ Anal. Calcd. for $\mathrm{C}_{10} \mathrm{H}_{10} \mathrm{~N}_{4} \mathrm{O}_{2} \mathrm{~S}_{2}$ Br: C, 62.12; H, 6.16; N, 18.14; S,7.23. Found: C, 65.78; H, 7.72; N, 14.37; S, 6.78. Yield: 78\% , M.P: $150^{\circ} \mathrm{C}$.

Table-1

\begin{tabular}{c|c|c|c|c|c|c|c|c|c}
\hline $\begin{array}{c}\text { Compound } \\
\text { No }\end{array}$ & $\mathrm{X}_{1}$ & $\mathrm{X}_{2}$ & $\mathrm{n}$ & $\mathrm{R}$ & $\mathrm{R}^{1}$ & Molecular Formula & $\begin{array}{c}\text { Molecular } \\
\text { Weight }\end{array}$ & Yield (\%) & $\mathrm{M} . \mathrm{P}\left({ }^{0} \mathrm{C}\right)$ \\
\hline VIa & $\mathrm{H}$ & $\mathrm{Br}$ & 2 & $\mathrm{CH}_{3}$ & $\mathrm{CH}_{3}$ & $\mathrm{C}_{14} \mathrm{H}_{19} \mathrm{BrN}_{4} \mathrm{O}$ & 340.80 & 69 & $142-144$ \\
\hline $\mathrm{VIb}$ & $\mathrm{H}$ & $\mathrm{Br}$ & 2 & $\mathrm{CH}_{3}$ & $\mathrm{C}_{2} \mathrm{H}_{5}$ & $\mathrm{C}_{16} \mathrm{H}_{23} \mathrm{BrN}_{4} \mathrm{O}$ & 367.28 & 72 & $154-156$ \\
\hline VIc & $\mathrm{H}$ & $\mathrm{Br}$ & 3 & $\mathrm{CH}_{3}$ & $\mathrm{CH}_{3}$ & $\mathrm{C}_{15} \mathrm{H}_{21} \mathrm{BrN}_{4} \mathrm{O}$ & 353.26 & 70 & $144-146$ \\
\hline VId & $\mathrm{H}$ & $\mathrm{Br}$ & 3 & $\mathrm{CH}_{3}$ & $\mathrm{C}_{2} \mathrm{H}_{5}$ & $\mathrm{C}_{17} \mathrm{H}_{25} \mathrm{BrN}_{4} \mathrm{O}$ & 381.31 & 62 & $178-180$ \\
\hline VIe & $\mathrm{H}$ & $\mathrm{Br}$ & 2 & $\mathrm{C}_{2} \mathrm{H}_{5}$ & $\mathrm{CH}_{3}$ & $\mathrm{C}_{16} \mathrm{H}_{23} \mathrm{BrN}_{4} \mathrm{O}$ & 367.28 & 74 & $182-184$ \\
\hline
\end{tabular}


RASĀYAN J. Chem.

Vol. 11 | No. 3 |1357 - 1365 | July - September | 2018

\begin{tabular}{c|c|c|c|c|c|c|c|c|c}
\hline VIf & $\mathrm{H}$ & $\mathrm{Br}$ & 2 & $\mathrm{C}_{2} \mathrm{H}_{5}$ & $\mathrm{C}_{2} \mathrm{H}_{5}$ & $\mathrm{C} 18 \mathrm{H} 27 \mathrm{BrN}_{4} \mathrm{O}$ & 395.34 & 72 & $134-136$ \\
\hline $\mathrm{VIg}$ & $\mathrm{H}$ & $\mathrm{Br}$ & 3 & $\mathrm{C}_{2} \mathrm{H}_{5}$ & $\mathrm{CH}_{3}$ & $\mathrm{C}_{17} \mathrm{H}_{25} \mathrm{BrN} 4 \mathrm{O}$ & 381.31 & 68 & $176-178$ \\
\hline VIh & $\mathrm{H}$ & $\mathrm{Br}$ & 3 & $\mathrm{C}_{2} \mathrm{H}_{5}$ & $\mathrm{C}_{2} \mathrm{H}_{5}$ & $\mathrm{C}_{19} \mathrm{H}_{29} \mathrm{BrN}_{4} \mathrm{O}$ & 409.36 & 66 & $156-158$ \\
\hline
\end{tabular}<smiles>[Y]c1cc([Y4])c(N)c(C(=O)O)c1</smiles><smiles>[X]c1cc([Y4])c2nc([SeH])n(CCN([R])P)c(=O)c2c1</smiles>

Dry Alcohol, $\mathrm{KOH}$

Dimethyl Sulfate, DMSO<smiles>[Y4]c1cc([Y2])c2nc(SC)n(C)c(=O)c2c1</smiles>

V
IsoPropyl alcohol, $\mathrm{HNR}_{2}{ }_{2}$

Scheme-1

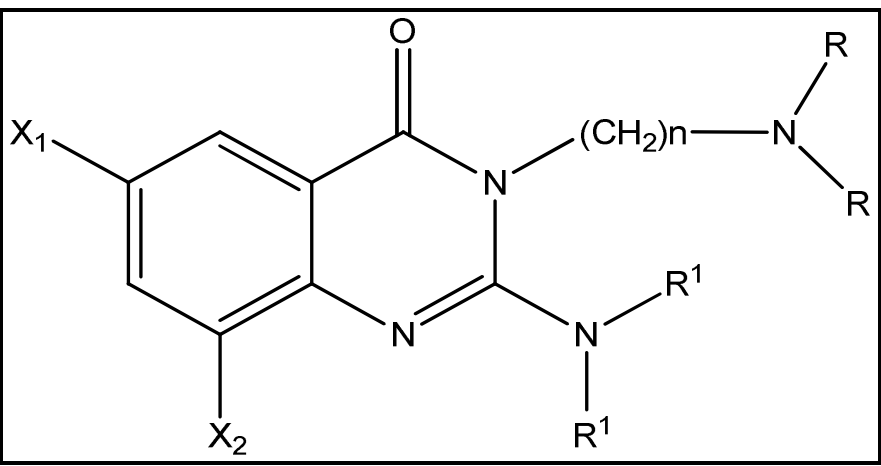

Compound VI<smiles>[X]c1cc([X])c2[nH]c(=S)n(C)c(=O)c2c1</smiles><smiles>[Y]c1cc([X])c2nc(N([R])[R])n(C)c(=O)c2c1</smiles>

VI (a-h) 
RASĀYAN J. Chem.

Vol. 11 | No. 3 |1357 - 1365 | July - September | 2018

\section{RESULTS AND DISCUSSION}

General Procedure for the Synthesis of 8-bromo-3-(2-(dimethylamino)ethyl)-2-thioxo-2,3Dihydroquinoline-4(1H)-one (III)

The crude product from the previous step (II; 0.01 mole) was taken and $15 \mathrm{ml}$ of dry alcohol was added. This reaction mixture was refluxed for 8 hours after the addition of appropriate alkyl amine (0.012 moles). The course of the reaction was monitored every hour by TLC. Completion of the reaction was confirmed by filter paper soaked in sodium nitroprusside failing to give pink color when exposed at the top of the condenser. Refluxing was continued until an unpleasant smell disappeared. Contents of the flask were concentrated under reduced pressure and poured into crushed ice. The product formed was filtered and dried to get 8-bromo-3-(2-(dimethylamino)ethyl)-2-thioxo-2,3- Dihydroquinoline-4(1H)-one (III).

IR $\left(\mathrm{cm}^{-1}\right) v_{\max }: 3340.2,3040.1,2983.3,1790.1,1670,1645,1590.9,1425,1375,845,700,{ }_{1}^{1} \mathrm{H}$ NMR (DMSO-d 6 , $300 \mathrm{MHz}) \delta: 2.27\left(\mathrm{~s}, 6 \mathrm{H},-\left(\mathrm{NCH}_{3}\right)_{2}\right), 2.66\left(\mathrm{t}, 2 \mathrm{H},-\mathrm{NCH}_{2}\right), 3.55\left(\mathrm{t}, 2 \mathrm{H},-\mathrm{NCH}_{2}\right), 4.0(\mathrm{~s}, 1 \mathrm{H},-\mathrm{NH}), 7.4-7.9(\mathrm{~m}, J=8.3$ $\mathrm{Hz}, 3 \mathrm{H}, \mathrm{Ar}-\mathrm{H}){ }^{13} \mathrm{C} \quad \mathrm{NMR}$ (DMSO-d $\left.6,75 \mathrm{MHz}\right) \delta: 45.6,53.9,55.5,121.4,127.4,129.2,130.4,135.4,141.5$, 161.5, 176.4,Mass: $m / z 327\left(\mathrm{M}^{+}\right), 329(\mathrm{M}+2)$,Anal. Calcd. for $\mathrm{C}_{12} \mathrm{H}_{14} \mathrm{~N}_{3} \mathrm{OSBr}: \mathrm{C}, 71.12 ; \mathrm{H}, 8.27 ; \mathrm{N}$, 12.75,S,4.24 Found: C, 64.23; H, 7.38; N, 13.57;S,5.79. Yield: $76 \%$, M.P: $164^{\circ} \mathrm{C}$.

General Method for the Synthesis of Potassium Salt of 8-bromo-3-(2-(dimethylamino)ethyl)-4-oxo1,2,3,4-tetrahydro quinazoline-2-thiolate (IV)

8-bromo-3-(2-(dimethylamino)ethyl)-2-thioxo-2,3- Dihydroquinoline-4(1H)-one (III 0.01 mole) was taken in dry alcohol $(10 \mathrm{ml})$ and potassium hydroxide $(0.015$ mole $)$ was added to it. This mixture was refluxed gently for $4 \mathrm{~h}$, until the product completely separated out. The progress of this reaction was monitored by TLC. The product is collected by decanting the liquid.

$\operatorname{IR}\left(\mathrm{cm}^{-1}\right) v_{\max }: \quad 3040.32,2923.16,2678.21,1790.35,1643.60,1541.18,1458.44,1219.29, \quad 1017.72,770.88,{ }^{1} \mathrm{H}$ NMR DMSO-d $6,300 \mathrm{MHz}) \delta: 2.27\left(\mathrm{~s}, 6 \mathrm{H},-\left(\mathrm{NCH}_{3}\right)_{2}\right), 2.66\left(\mathrm{t}, 2 \mathrm{H},-\mathrm{NCH}_{2}\right), 3.55\left(\mathrm{t}, 2 \mathrm{H},-\mathrm{NCH}_{2}\right), 7.4-7.9(\mathrm{~m}, J=$ $8.4 \mathrm{~Hz}, 3 \mathrm{H}, \mathrm{Ar}-\mathrm{H}$ ). ${ }^{13} \mathrm{C} \quad \mathrm{NMR}$ (DMSO-d 6 ,75 MHz) $\delta: ~ 45.6,49.5,55.6,111.2,121.4,127.4,129.2,135.4$, 161.5,163.5,Mass: $\mathrm{m} / \mathrm{z}$ 346(M+),348(M+2),Anal. Calcd. for $\mathrm{C}_{12} \mathrm{H}_{13} \mathrm{~N}_{3} \mathrm{OSBrK}$ : C, 74.91; H, 9.71; N, 9.85; Found: C, 70.39; H,8.58;N,10.18. Yield: $73 \%$, M.P: $156^{\circ} \mathrm{C}$.

General Procedure for the Synthesis of 8-bromo-3-(2-(dimethylamino)ethyl)-2(methylthio)quinazoline-4(3H)-one (Va-h)

The potassium salt (IV; 0.01 mole) was dissolved in $10 \mathrm{ml}$ of dimethyl sulphoxide and cooled to $0-10^{\circ} \mathrm{C}$. Dimethyl sulphate (0.01 mole) was added slowly in a drop-wise manner. Stirring was continued for 4 hours at $15-20^{\circ} \mathrm{C}$. This was poured into crushed ice and extracted with chloroform. This was dried over anhydrous sodium sulphate. The chloroform extract was evaporated to get 8-bromo-3-(2(dimethylamino)ethyl)-2-(methylthio)quinazolin-4(3H)-one. (Va-h).

Va: $\quad \operatorname{IR}\left(\mathrm{cm}^{-1}\right) v_{\max }: 3040.32,2923.16,2990.25,2678.21,1790.35,1643.60,1541.18,1458.44,1219.29$, 1017.72, 770.88, ${ }^{1} \mathrm{H}$ NMR (DMSO-d $\left.6,300 \mathrm{MHz}\right) \delta: 1.82\left(\mathrm{~s}, 3 \mathrm{H}, \mathrm{S}-\mathrm{CH}_{3}\right), 2.27\left(\mathrm{~s}, 6 \mathrm{H},-\left(\mathrm{NCH}_{3}\right)_{2}\right), 2.66(\mathrm{t}, 2 \mathrm{H},-$ $\left.\mathrm{NCH}_{2}\right), 3.55\left(\mathrm{t}, 2 \mathrm{H},-\mathrm{NCH}_{2}\right), 7.4-7.9(\mathrm{~m}, \quad J=8.4 \mathrm{~Hz}, 3 \mathrm{H}, \mathrm{Ar}-\mathrm{H}),{ }^{13} \mathrm{C} \quad \mathrm{NMR}\left(\mathrm{DMSO}_{6} \mathrm{~d}_{6}, 75 \mathrm{MHz}\right) \delta$ : 37.5,45.6,47.2,49.5,55.6,111.2,121.4,127.4,129.2,135.4, 161.5,163.5, Mass: $m / z$ 341( $\left.\mathrm{M}^{+}\right), 343(\mathrm{M}+2)$.

Anal. Calcd. for $\mathrm{C}_{13} \mathrm{H}_{16} \mathrm{~N}_{3} \mathrm{OSBr}$ : C, 62.59; H,9.25; N,9.19;S,4.31; Found: C, 60.17; H, 6.91; N, 9.01;S,5.28. Yield: $68 \%$, M.P: $172^{\circ} \mathrm{C}$.

General Procedure for the Synthesis of 2-N, N-dialkyl amino 3-(N, N-dialkylamino) alkyl-6bromo-2-methylthio-quinazoline-4(3H)-ones (VI)

8-bromo-3-(2-(dimethylamino)ethyl)-2-(methylthio)quinazoline-4(3H)-one $\quad(\mathbf{V} ; \quad 0.01$ mole) was dissolved in $10 \mathrm{ml}$ of isopropyl alcohol and dialkylamine $(0.015 \mathrm{~mole})$ was added. This reaction mixture was refluxed till the unpleasant smell disappeared. The reaction mixture was poured into crushed ice. The product formed was filtered and recrystallized from isopropyl alcohol to get 2-N, N-dialkyl amino 3-(N, N-dialkylamino) alkyl-6-bromo-2-methylthio-quinazoline-4(3H)-ones (VI a-h). 
VIa: 8-bromo-2-(dimethylamino)-3-(2-(dimethylamino)ethyl)quinazolin-4(3H)-one IR $\left(\mathrm{cm}^{-1}\right) v_{\max }: 3040.32,2998.25,2925.10,2678.21,1790.35,1643.60,1017.72,770.85,{ }^{1} \mathrm{H}$ NMR $\left(\mathrm{DMSO}_{6} \mathrm{~d}_{6}, 300 \mathrm{MHz}\right) \delta: 1.80\left(\mathrm{~s}, 6 \mathrm{H}, \mathrm{N}-\left(\mathrm{CH}_{3}\right)_{2}\right), 2.27\left(\mathrm{~s}, 6 \mathrm{H},-\left(\mathrm{NCH}_{3}\right)_{2}\right), 2.66\left(\mathrm{t}, 2 \mathrm{H},-\mathrm{NCH}_{2}\right), 3.55\left(\mathrm{t}, 2 \mathrm{H},-\mathrm{NCH}_{2}\right)$, $\begin{array}{llll}7.4-7.9 & (\mathrm{~m}, \quad \mathrm{~J} & 8.2 \quad \mathrm{~Hz}, & 3 \mathrm{H}, \mathrm{Ar}-\mathrm{H}),{ }^{13} \mathrm{CNMR}\left(\mathrm{DMSO}-\mathrm{d}_{6}, 75 \mathrm{MHz}\right)\end{array}$ $\delta: 40.2,43.3,47.1,48.4,49.1,54.3,127.4,129.2,130.4,131.4,132.5,150.3,159.3,171.2 . M a s s: ~ m / z ~ 340.8\left(\mathrm{M}^{+}\right)$, 342.8(M+2),Anal. Calcd. for $\mathrm{C}_{14} \mathrm{H}_{19} \mathrm{BrN}_{4} \mathrm{O}: \mathrm{C}, 59.12 ; \mathrm{H}, 8.59 ; \mathrm{N}, 14.65$; Found: C, 58.17; H, 8.95; N, 12.02 .

VIb: 8-bromo-2-(diethylamino)-3-(2-(dimethylamino)ethyl)quinazolin-4(3H)-one

IR $\left(\mathrm{cm}^{-1}\right) \quad v_{\max }: 3040.32,2998.25,2925.10,2678.21,1790.35,1643.60,1017.72,770.85,{ }^{1} \mathrm{H}$ NMR $\left(\mathrm{DMSO}_{6} \mathrm{~d}_{6}, \quad 300 \mathrm{MHz}\right) \quad \delta: \quad 1.74\left(\mathrm{~s}, 6 \mathrm{H},\left(\mathrm{CH}_{3}\right)_{2}\right), \quad 1.80\left(\mathrm{t}, 6 \mathrm{H},\left(\mathrm{CH}_{3}\right)_{2}\right), 1.87\left(\mathrm{q}, 4 \mathrm{H},\left(\mathrm{CH}_{2}\right)_{2}, \quad 2.60(\mathrm{t}, 2 \mathrm{H},-\right.$ $\left.\mathrm{NCH}_{2}\right), 3.55\left(\mathrm{t}, 2 \mathrm{H},-\mathrm{NCH}_{2}\right), \quad 7.4-7.9(\mathrm{~m}, \quad J=8.2 \mathrm{~Hz}, \quad 3 \mathrm{H}, \mathrm{Ar}-\mathrm{H}),{ }^{13} \mathrm{CNMR}\left(\mathrm{DMSO}-\mathrm{d}_{6}, 75 \mathrm{MHz}\right)$ $\delta: 38.2,41.3,46.3,46.2,46.0,52.3,127.4,129.2,130.4,131.4$,

132.5, 150.3, 159.3, 171.2,Mass: $\mathrm{m} / \mathrm{z}$ 367.2 $\left(\mathrm{M}^{+}\right)$, 367.2(M+2),Anal. Calcd. for $\mathrm{C}_{16} \mathrm{H}_{23} \mathrm{BrN} 4 \mathrm{O}: \mathrm{C}, 60.12$; $\mathrm{H}, 8.12$; N,12.55; Found: C, 58.20; H, 8.05; N, 12.22 .

VIc: 8-bromo-2-(dimethylamino)-3-(3-(dimethylamino)propyl)quinazolin-4(3H)-one IR $\left(\mathrm{cm}^{-1}\right) v_{\max }: 3032.12,2990.25,2885.3,2653.21,17850.35,1625.60,1010.72,765.85,{ }^{1} \mathrm{H}$ NMR (DMSO-d 6 , $300 \mathrm{MHz}) \delta: 1.80\left(\mathrm{~s}, 6 \mathrm{H}, \mathrm{N}-\left(\mathrm{CH}_{3}\right)_{2}\right), 2.27\left(\mathrm{~s}, 6 \mathrm{H},-\left(\mathrm{NCH}_{3}\right)_{2}\right), 2.66\left(\mathrm{t}, 2 \mathrm{H},-\mathrm{NCH}_{2}\right), 2.87\left(\mathrm{~m}, 2 \mathrm{H},-\mathrm{CH}_{2}\right), 3.55(\mathrm{t}, 2 \mathrm{H},-$ $\left.\mathrm{NCH}_{2}\right), 4.0(\mathrm{~s}, 1 \mathrm{H},-\mathrm{NH}), 7.4-7.9(\mathrm{~m}, \quad J=8.2 \mathrm{~Hz}, \quad 3 \mathrm{H}, \mathrm{Ar}-\mathrm{H}),{ }^{13} \mathrm{CNMR}\left(\mathrm{DMSO}-\mathrm{d}_{6}, 75 \mathrm{MHz}\right)$ $\delta: 40.2,43.3,47.1,48.4,49.1,54.3,56.2,127.4,129.2,130.4,131.4,132.5,150.3,159.3,171.2$, Mass: $\mathrm{m} / \mathrm{z} \quad 353.28$ $\left(\mathrm{M}^{+}\right), 355.8(\mathrm{M}+2)$,Anal. Calcd. for $\mathrm{C}_{15} \mathrm{H}_{21} \mathrm{BrN}_{4} \mathrm{O}$ : C,71.31; H,9.29; N,12.65; Found: C, 68.17; H, 9.95; N, 11.02 .

VId: 8-bromo-2-(diethylamino)-3-(3-(dimethylamino)propyl)quinazolin-4(3H)-one

IR $\left(\mathrm{cm}^{-1}\right) v_{\max }: 3040.32,2998.25,2925.10,2678.21,1790.35,1643.60,1017.72,770.85,{ }^{1} \mathrm{H} \mathrm{NMR}$ $\left(\mathrm{DMSO}_{6}, 300 \mathrm{MHz}\right) \delta: 1.72\left(\mathrm{~s}, 6 \mathrm{H}, \mathrm{N}-\left(\mathrm{CH}_{3}\right)_{2}\right), 2.25\left(\mathrm{t}, 6 \mathrm{H},-\left(\mathrm{NCH}_{3}\right)_{2}\right), 2.40\left(\mathrm{q}, 4 \mathrm{H}, \mathrm{N}-\mathrm{CH}_{2}\right)_{2}, 2.60\left(\mathrm{t}, 2 \mathrm{H},-\mathrm{NCH}_{2}\right)$, $2.82\left(\mathrm{~m}, 2 \mathrm{H},-\mathrm{CH}_{2}\right), 3.52\left(\mathrm{t}, 2 \mathrm{H},-\mathrm{NCH}_{2}\right), \quad 7.4-7.9(\mathrm{~m}, \quad J=8.2 \mathrm{~Hz}, 3 \mathrm{H}, \mathrm{Ar}-\mathrm{H}),{ }^{13} \mathrm{CNMR}\left(\mathrm{DMSO}-\mathrm{d}_{6}, 75 \mathrm{MHz}\right)$ $\delta: 38.2,41.3,46.1,46.4,48.1,52.3,121.4,124.2,128.4,130.4,131.5,145.3,156.3,170.2$, Mass: $\mathrm{m} / \mathrm{z} 381.31$ $\left(\mathrm{M}^{+}\right), 383.3(\mathrm{M}+2)$,Anal. Calcd. for $\mathrm{C}_{17} \mathrm{H}_{25} \mathrm{BrN}_{4} \mathrm{O}$ : C,74.12; H,8.01; N,12.35; Found: C, 69.17; H, 9.95; $\mathrm{N}, 10.02$.

VIe: 8-bromo-2-(dimethylamino)-3-(2-(diethylamino)ethyl)quinazolin-4(3H)-one

IR $\left(\mathrm{cm}^{-1}\right) v_{\max }: 3032.32,2908.25,2901.10,2651.21,1775.35,1640.60,1010.72,765.85,{ }^{1} \mathrm{H}$ NMR (DMSO$\left.\mathrm{d}_{6}, 300 \mathrm{MHz}\right) \delta: 1.70\left(\mathrm{t}, 6 \mathrm{H},\left(\mathrm{CH}_{3}\right)_{2}\right), 1.86\left(\mathrm{q}, 4 \mathrm{H}, \mathrm{N}-\mathrm{CH}_{2}\right)_{2}, 2.26\left(\mathrm{~s}, 6 \mathrm{H},-\left(\mathrm{NCH}_{3}\right)_{2}\right), 2.62\left(\mathrm{t}, 2 \mathrm{H},-\mathrm{NCH}_{2}\right), 3.53(\mathrm{t}, 2 \mathrm{H},-$

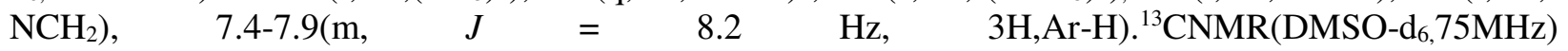
$\delta: 38.2,39.1,42.3,46.1,46.4,48.1,53.3,126.4,128.2,131.4,130.4,131.5,149.3,158.3,169.2$, Mass: $\mathrm{m} / \mathrm{z} \quad 361.38$ $\left(\mathrm{M}^{+}\right), 363.38(\mathrm{M}+2)$,Anal. Calcd. for $\mathrm{C}_{16} \mathrm{H}_{23} \mathrm{BrN}_{4} \mathrm{O}$ : C,76.12; H,9.36; N,11.01;Found: C, 74.17; H, 9.90; N, 10.01 .

VIf: 8-bromo-2-(diethylamino)-3-(2-(diethylamino)ethyl)quinazolin-4(3H)-one IR $\left(\mathrm{cm}^{-1}\right) v_{\max }: 3040.32,2938.25,2928.10,2688.21,1771.35,1621.60,1017.72,770.85,{ }^{1} \mathrm{H}$ NMR (DMSO$\left.\mathrm{d}_{6}, 300 \mathrm{MHz}\right) \delta: 1.65\left(\mathrm{t}, 6 \mathrm{H},\left(\mathrm{CH}_{3}\right)_{2}\right), 1.86\left(\mathrm{q}, 4 \mathrm{H}, \mathrm{N}-\mathrm{CH}_{2}\right)_{2}, 2.26\left(\mathrm{t}, 6 \mathrm{H},-\left(\mathrm{CH}_{3}\right)_{2}\right), 2.86\left(\mathrm{q}, 4 \mathrm{H}, \mathrm{N}-\mathrm{CH}_{2}\right)_{2}, 3.25(\mathrm{t}, 2 \mathrm{H},-$ $\left.\mathrm{NCH}_{2}\right), \quad 3.55\left(\mathrm{t}, 2 \mathrm{H},-\mathrm{NCH}_{2}\right), \quad 7.4-7.9(\mathrm{~m}, \quad J=8.2 \mathrm{~Hz}, \quad 3 \mathrm{H}, \mathrm{Ar}-\mathrm{H}),{ }^{13} \mathrm{CNMR}(\mathrm{DMSO}-$

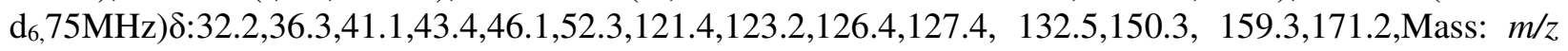
$395.3\left(\mathrm{M}^{+}\right), 397.8(\mathrm{M}+2)$,Anal. Calcd. for $\mathrm{C}_{18} \mathrm{H}_{27} \mathrm{BrN}_{4} \mathrm{O}$ : C,76.31; $\mathrm{H}, 10.12 ; \mathrm{N}, 10.12$; Found: C, 72.17; $\mathrm{H}$, $10.95 ; \mathrm{N}, 10.02$.

VIg: 8-bromo-3-(3-(diethylamino)propyl)-2-(dimethylamino)quinazolin-4(3H)-one IR $\left(\mathrm{cm}^{-1}\right) v_{\max }: 3021.32,2978.25,2915.10,2628.21,1760.35,1623.60,1005.72,770.85,{ }_{1}^{1} \mathrm{H}$ NMR (DMSO-d 6 , $300 \mathrm{MHz}) \quad \delta: 1.65\left(\mathrm{t}, 6 \mathrm{H},\left(\mathrm{CH}_{3}\right)_{2}\right), 1.86\left(\mathrm{q}, 4 \mathrm{H}, \mathrm{N}-\mathrm{CH}_{2}\right)_{2}, 2.26\left(\mathrm{t}, 6 \mathrm{H},-\left(\mathrm{CH}_{3}\right)_{2}\right), 2.86\left(\mathrm{q}, 4 \mathrm{H}, \quad \mathrm{N}-\mathrm{CH}_{2}\right)_{2}$, 
RASĀYAN J. Chem.

Vol. 11 | No. 3 |1357 - 1365 | July - September | 2018

$3.20\left(\mathrm{~m}, 2 \mathrm{H}, \mathrm{CH}_{2}\right), 3.55\left(\mathrm{t}, 2 \mathrm{H},-\mathrm{NCH}_{2}\right), 7.4-7.9(\mathrm{~m}, \quad J=8.2 \mathrm{~Hz}, 3 \mathrm{H}, \mathrm{Ar}-\mathrm{H}),{ }^{13} \mathrm{CNMR}\left(\mathrm{DMSO}-\mathrm{d}_{6}, 75 \mathrm{MHz}\right)$ $\delta: 40.2,43.3,47.1,48.4,49.1,54.3,127.4,129.2,130.4,131.4,132.5,150.3,159.3,171.2$, Mass: $\quad m / z \quad 381.31$ $\left(\mathrm{M}^{+}\right)$,383.3(M+2),Anal. Calcd. for $\mathrm{C}_{17} \mathrm{H}_{25} \mathrm{BrN}_{4} \mathrm{O}$ : C,59.12; H,8.59; N,14.65; Found: C, 58.17; H, 8.95; N, 12.02 .

VIh: 8-bromo-3-(3-(diethylamino)propyl)-2-(diethylamino)quinazolin-4(3H)-one

IR $\left(\mathrm{cm}^{-1}\right) v_{\max }: 3040.32,2998.25,2925.10,2678.21,1790.35,1643.60,1017.72,770,{ }^{1} \mathrm{H}$ NMR (DMSO-d 6,300 $\mathrm{MHz}) \quad \delta: \quad 1.60\left(\mathrm{t}, 6 \mathrm{H},\left(\mathrm{CH}_{3}\right)_{2}\right), 1.75\left(\mathrm{q}, 4 \mathrm{H}, \mathrm{N}-\mathrm{CH}_{2}\right)_{2}, 2.20\left(\mathrm{t}, 6 \mathrm{H},-\left(\mathrm{CH}_{3}\right)_{2}\right), 2.80(\mathrm{q}, 4 \mathrm{H}, \quad \mathrm{N}-$ $\left.\mathrm{CH}_{2}\right)_{2}, 3.20\left(\mathrm{~m}, 2 \mathrm{H}, \mathrm{CH}_{2}\right), 3.55\left(\mathrm{t}, 2 \mathrm{H},-\mathrm{NCH}_{2}\right), 3.67\left(\mathrm{t}, 2 \mathrm{H},-\mathrm{NCH}_{2}\right), 7.4-7.9(\mathrm{~m}, \quad J=8.2 \mathrm{~Hz}, 3 \mathrm{H}, \mathrm{Ar}-$ $\mathrm{H}),{ }^{13} \mathrm{CNMR}\left(\mathrm{DMSO}_{6} \mathrm{~d}_{6}, 75 \mathrm{MHz}\right) \quad \delta: 32.2,40.3,42.1,44.4,47.1,52.3,125.4,127.2,128.4,130.4$, 131.5,150.3,159.3,171.2.Mass: $\mathrm{m} / z$ 409.3 $\left(\mathrm{M}^{+}\right), 411.3(\mathrm{M}+2)$,Anal. Calcd. for $\mathrm{C}_{19} \mathrm{H}_{29} \mathrm{BrN}_{4} \mathrm{O}: \mathrm{C}, 72.92$; H,10.59; N,13.65; Found: C, 70.16; H, 10.95; N, 10.02 .

\section{Anti-Microbial Activity}

The literature survey has revealed that 2-N, N-dialkyl amino 3-(N, N-dialkylamino) alkyl-6-bromo-2methylthio-quinazoline-4(3H)-ones derivatives are well-known exhibits their wide range of biological activities. It is, therefore, considered worthwhile to evaluate the present Quinazoline derivatives of different types synthesized and their possible antimicrobial activities. The antimicrobial activity was determined by using disc diffusion method by measuring the zone of inhibition in $\mathrm{mm}$. All the compounds, (a-h) were screened in-vitro at a concentration of $50 \mu \mathrm{g} / \mathrm{mL}$ for antibacterial activity against three Gram-positive Bacillus subtilis(B. subtilis), Bacillus sphaericus(B.sphaericus) and Staphylococcus aureus(S.aureus), and three Gram-negative bacteria viz. Pseudomonas aeruginosa(P.aeruginosa), Klebsiella aerogenesa(K. aerogenesa) and Chromobacterium violaceum(C.violaceum). Standard antibacterial drug SM (Streptomycin), $(50 \mu \mathrm{g} /$ disc) was also tested under similar conditions against these organisms. Each experiment was done in triplicate and the average reading was taken. Most of the compounds were found to display well to moderate antibacterial activity against different strains of bacteria. From the Table-3, it was observed that among all the compounds tested, sulphonyl derivatives $\mathbf{2 a}, \mathbf{2 c}, \mathbf{2 e}$, and $\mathbf{2 f}$ showed good to excellent activity, while the remaining derivatives showed moderate activity against all the tested bacterial strains. The results of antibacterial activity and antifungal activity are expressed in Table-3 and Table-4. An attempt has also been made to correlate their structure and activity in view of the presence of different substituents.

Table 2: Antibacterial Activity of Compounds VI(a-h).

\begin{tabular}{c|c|c|c|c|c|c}
\hline \multirow{2}{*}{ Compd. } & \multicolumn{6}{|c}{ Minimum Inhibitory Concentration (MIC) in $\mu \mathrm{g} / \mathrm{mL}$} \\
\cline { 2 - 7 } & B. subtilis & B. sphaericus & S. aureus & $\begin{array}{l}\text { P. } \\
\text { aeruginosa }\end{array}$ & $\begin{array}{l}\text { K. } \\
\text { aerogenes }\end{array}$ & C. violaceum \\
\hline VIa & 32 & 30 & 30 & 28 & 28 & 30 \\
\hline VIb & 20 & 24 & 24 & 22 & 24 & 28 \\
\hline VIc & 38 & 34 & 32 & 30 & 28 & 36 \\
\hline VId & 18 & 20 & 18 & 24 & 26 & 26 \\
\hline VIe & 28 & 30 & 32 & 28 & 32 & 32 \\
\hline VIf & 32 & 34 & 34 & 36 & 32 & 30 \\
\hline VIg & 20 & 22 & 24 & 22 & 24 & 26 \\
\hline VIh & 22 & 24 & 24 & 22 & 24 & 26 \\
\hline SM & 30 & 28 & 30 & 32 & 30 & 28 \\
\hline SM = Streptomycin. & \multicolumn{7}{l}{}
\end{tabular}

\section{Antibacterial Activity}

The in vitro antibacterial activity 2-N, N-dialkyl amino 3-(N, N-dialkylamino) alkyl-6-bromo-2methylthio-quinazoline-4(3H)-ones VI(a-h) was assessed against three representative Gram-positive 
bacteria viz. Bacillus subtilis, Bacillus sphaericus and Staphylococcus aureus, and three Gram-negative bacteria viz. Pseudomonas aeruginosa, Klebsiella aerogenes and Chromobacterium violaceum by the broth dilution method recommended by the National Committee for Clinical Laboratory Standards. Bacteria were grown overnight in Luria Bertani (LB) broth at $37^{\circ} \mathrm{C}$, harvested by centrifugation and then washed twice with sterile distilled water. Stock solutions of the series of compounds were prepared in DMSO. Each stock solution was diluted with standard method broth (Difco) to prepare serial two-fold dilutions in the range of 50 to $0.8 \mu \mathrm{g} / \mathrm{mL}$. Ten micro liters of the broth containing about $10^{5}$ colonyforming units $(\mathrm{cfu}) / \mathrm{mL}$ of test bacteria were added to each well of a 96 -well micro titer plate. Culture plates were incubated for $24 \mathrm{~h}$ at $37^{\circ} \mathrm{C}$, and the growth of bacteria was monitored by visually and spectrophotometrically. Penicillin and Streptomycin were also screened under identical conditions for comparison. The obtained data of compounds VI(a-h) are presented in Table-3. It has been observed that the compounds exhibit interesting biological activity, however, with a degree of variation.In the series of VI(a-h), the compounds VIa, VIc, VIe and VIf are found to be the most active against Gram-positive bacteria and the Gram-negative bacteria.The remaining compounds showed moderate to good activity against all the Gram-positive bacteria and the Gram-negative bacteria.

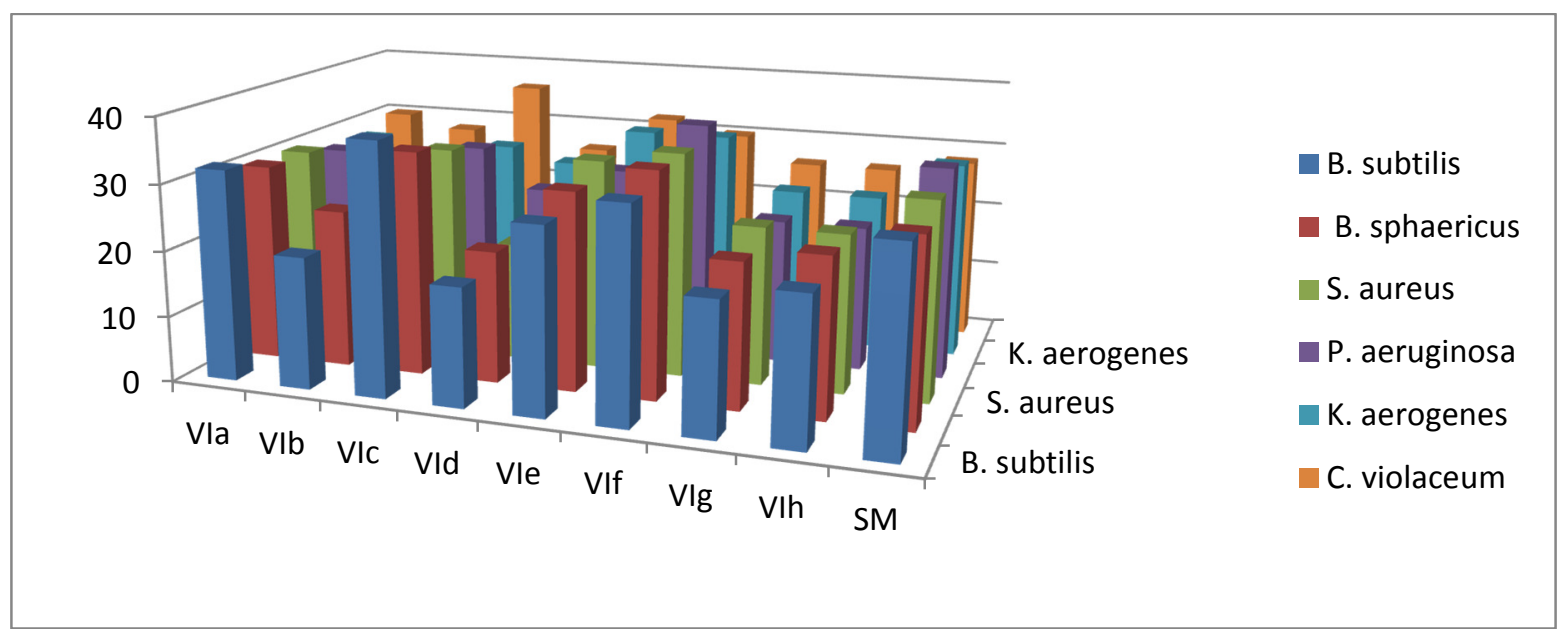

\section{Antifungal Activity}

Fig.-1: a Graphical form of Anti-Bacterial Screening

The compounds VI(a-h) were also screened for their antifungal activity against Candida albicans (C.albicans)(ATCC 10231), Aspergillus fumigates(A.fumigatus) (HIC 6094), Trichophyton rubrum(T. rubrum) (IFO 9185), and Trichophyton mentagrophytes(T. mentagrophytes) (IFO 40996) in dimethyl sulfoxide (DMSO) by disc diffusion method. Amphotericin B was used as a standard drug and the mean inhibition zone (MZI) data were measured and compared with controls, the MZI values of the compounds screened are given in Table-4.The antifungal screening data showed the appreciable activity of the test compounds. Among the screened compounds, compound VIa, VIc and VIg showed good antifungal activity. Remaining compounds showed moderate antifungal activity against test compounds.

Table-3: Antifungal Activity of Compounds VI(a-h)

\begin{tabular}{c|c|c|c|c}
\hline \multirow{2}{*}{ Compnd } & \multicolumn{4}{|c}{ Mean zone inhibition (MZI) ${ }^{\mathrm{a}}$ in $10 \mu \mathrm{g} / \mathrm{mL}$} \\
\cline { 2 - 5 } & C. albicans & A. fumigatus & T. rubrum & T. mentagropytes \\
\hline VIa & 28 & 28 & 26 & 26 \\
\hline VIb & 18 & 20 & 18 & 20 \\
\hline VIc & 28 & 32 & 30 & 28 \\
\hline VId & 20 & 22 & 26 & 24 \\
\hline
\end{tabular}


RASĀYAN J. Chem.

Vol. 11 | No. 3 |1357 - 1365 | July - September | 2018

\begin{tabular}{c|c|c|c|c}
\hline VIe & 22 & 26 & 24 & 18 \\
\hline VIf & 18 & 20 & 22 & 24 \\
\hline VIg & 30 & 32 & 32 & 28 \\
\hline VIh & 24 & 26 & 21 & 20 \\
\hline Amphotericin B & 26 & 28 & 28 & 26 \\
\hline
\end{tabular}

${ }^{\mathrm{a}}$ Values are mean $(\mathrm{n}=3)$.

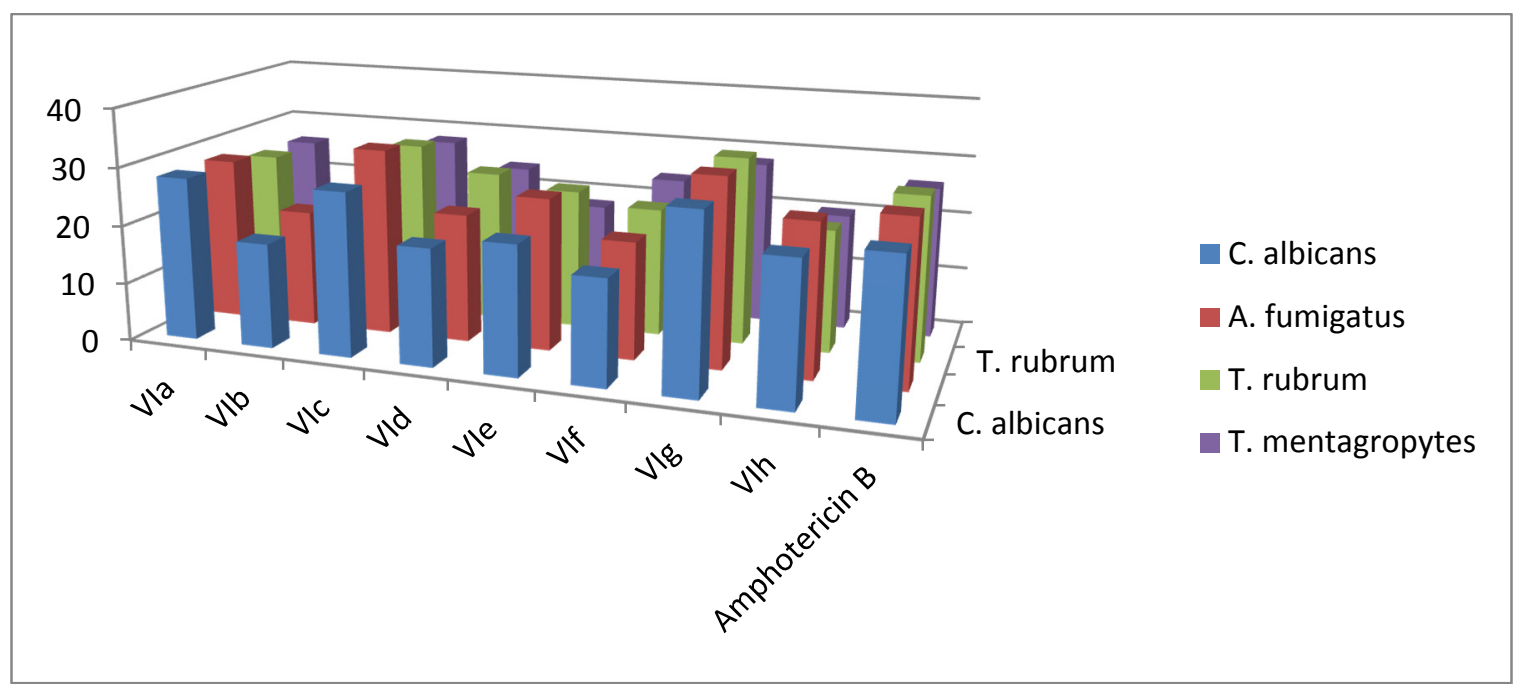

Fig.-2: a Graphical form of Anti-Fungal Screening

Inhibition Zone in mm (--indicates no inhibitory activity)Control inhibition zone (which indicates inhibition zone of solvent) was subtracted from the inhibition zone of compounds which gives actual inhibition zone of compounds.

\section{CONCLUSION}

In conclusion, a series of quinazolinone derivatives VI(a-h) were prepared. The antibacterial activity of these compounds was evaluated against various bacteria's; antifungal activity was evaluated against various fungi. The compounds showed a variable degree of antimicrobial activity. Among the screened compounds, VIc, VIf and VIg were found to be the most active against all the microorganisms employed both for antibacterial and antifungal activity. With this set of analogs, we are now in a position to investigate the multiple biological activities of these compounds.

\section{ACKNOWLEDGMENT}

The author and Co-author are gratefully thanking the Head, Department of Pharmacy \& Biotechnology, for providing necessary laboratory facilities, We also thankful to Head, Department of Chemistry, Osmania University, We also grateful thanks to Director, Indian Institute of Chemical Technology, Hyderabad, India, for providing NMR,CMR,IR and Mass spectral data. Finally we thankful to Head, Department of Biotechnology, Osmania University, India, for Biological evaluation studies.

\section{REFERENCES}

1. L. F. Miliani, O. H. Nielsen, P. S. Andersen, S. E. Girardin, Clin.Exp.Immunol.,147, 227(2006).

2. G. Ravi, A. Ravinder Nath, A. Nagaraj, Journal of Current Chemical \& Pharmaceutical Sciences, 4(3),126(2014).

3. A. Salminen, J. M .T. Hyttinen, K. Kaarniranta, J.Mol.Med, 89,667(2011).

4. G. Ravi, A. Ravinder Nath, A. Nagaraj, S. Damodhar. G. Nageshwara Rao, Derpharma Chemica, 6(4), 223(2014).

5. T. Lawrence, The Nuclear Factor NF-kB Pathway in Inflammation,16,26(1985). 
RASĀYAN J. Chem.

Vol. 11 | No. 3 |1357 - 1365 | July - September | 2018

6. Cold Spring Harb.Perspect.Biol,1651,(2009).

7. C. A. Dinarello, Annu.Rev.Immunol, 27,519(2009).

8. F. R. Greten, M. C. Arkan, J. Bollrath, L. C. Hsu, J. Goode, C. Miething, S. I. Goktuna, M. Neuenhahn, J. Fierer, S. Paxian, N. VanRooijen, Y. Xu, T. O'Cain, B. B. Jaffee, D. H. Busch, J. Duyster, R. J. Schmid, L. Eckmann, M. Karin, Pharmacological Inhibition of IKK b.Cel,130,918(2007).

9. T. Lawrence, D.W.Gilroy, P. Colville-Nash, R. Willoughby,Nat.Med,7,1291(2001).

10. H.K.Eltzschig,P.Carmeliet,N.Engl.J.Med,364,656(2011).

11. S.Ohki,N.Ogino,S.Amamoto,O.Hayaishi, J. Biol. Chem,254,829(1979).

12. A. Nagaraj, G. Ravi, S. Sharath Kumar Goud, S. Raghaveer, Neelofer Rana, Research and Reviews: Journal of Chemistry, 3,17(2014).

13. S. Damodhar, Guguloth Ravi, A. Ravinder Nath, Der Pharmacia Sinica,7(1),16(2016).

14. (a)R. Merchant, K. Jayasukhal, D. S. Chothia, J. Med. Chem., 13,335(1976), (b)M. S. Shingare, D. V. Ingale, J. Med. Chem.,53,1036(1976).

15. J. S. Goes, A. Alexandre, P. Romulo, C. Tenorio, S. Cristione, B. Carvalho, S. Carla, B. Pessanha, G. Jose, A. Delima, R. Antonio, A. Farioa, J. Antonio, E. Alves, Bioorg.Med.Chem.Lett.,15,2575(2005).

16. J. Blanchet, J. Zhu, Tetrahedron Lett., 45,4449(2004).

17. K. A. Kandeel, Arkivoc, 1 (2006).

18. S. G. Kucukguzel, E. E. Oruc, S. Rollas, F. Sahin, A. Ozbek, Eur. J. Med. Chem., 37,197(2002).

19. T. Mizutani, T. Nagase, S. Ito, Y. Miyamoto, T. Tanaka, N. Take-naga, S. Tokita, N. Sato, Bioorg. Med.Chem Lett,18, 6041(2008).

20. K. S. Prasad, L. S. Kumar, S. Chandan, B. Jayalakshmi, Revanasiddappaa, Spectrochim. Acta.A., 81, 276(2011).

21. B. Junne, S. Wadje, V. Baig, B. Vibhute, Int. J. Chem. Sci., 5,2093(2007).

22. C. Ling, T. Jing, W. Coi, W. J. Duo-Zui, Chin, Chem. Soc., 53,1193(2006).

23. (a)A. Srinivas, A. Nagaraj, Ch. Sanjeeva Reddy, Eur. J. Med. Che.,45,2353(2010), (b)Ch. Sanjeeva Reddy, D. C. Rao, V. Yakub, A. Nagaraj, Chem.Pharm.Bull., 58,805(2010), (c)Ch. Sanjeeva Reddy, A. Srinivas, A.Nagaraj, Chem. Pharm. Bull., 57,685(2009)

24. H. Hasegawa, M. I. Muraoka, K. Matsuia, A. Kojima, Bioorg.Med.Chem.Let., 16,727(2006).

25. R. A. Friesner, J. L. Banks, R. B. Murphy, T. A. Halgren, J. J. Klicic, D. T. Mainz, M. P. Repasky, E. H. Knoll, M. Shelley, J. K. Perry, D. E. Shaw, P. Francis, P. S. Shenkin, J. Med. Chem.,47,1739(2004).

26. T. Hou, J. Wang,Y. Li, W.Wang, J. Chem. Inf. Model, 51, 69(2011).

27. P. D. Lyne, M. L. Lamb, J. C. Saeh, J. Med. Chem,49,4805(2006).

28. Qikprop, Schrodinger, LLC, NY, 8(2011).

29. A.Cavalli, E. Poluzzi, F. D.Ponti, M. Recanatini, J. Med. Chem., 45, 3844(2002).

30. A. Nagaraj, G. Ravi, G. NageshwaraRao, S. Sharath Kumar Goud, Naseem, Organic Communications, 5,160(2012).

31. R. Ottana, R. Maccari, M. L. Barreca, G. Bruno, A. Rotondo, A. Rossi, G. Chiricosta, D. Paola, L.Sautebin,S.Cuzzocrea,M.Vigorita,G.Giorg.Med.Chem.,13,4243(2005).

32. P. Chaitanya, G. Deepak Reddy, G. Varun, L. M. Srikanth, V. V. S. R. Prasad, A. Ravindernath, MedicinalChemistry, 10,711(2014).

[RJC-1599/2017] 